\title{
A frequency independent criterion for describing sound absorbing materials by
}

\section{a limp frame model}

Olivier Doutres, Nicolas Dauchez, ${ }^{*}$ Jean-Michel Génevaux, and Olivier Dazel

LAUM, CNRS, Université du Maine, Av. O. Messiaen, 72095 LE MANS,

France

(Dated: October 22, 2008)

Authors' accepted manuscript. The archived file is not the final published version of the article.

Olivier Doutres, Nicolas Dauchez, Jean-Michel Génevaux, Olivier Dazel. "A frequency independent criterion for describing sound absorbing materials by a limp frame model," in: Acta Acustica united with Acustica, vol. 95, 2009, p. 178-181. http://dx.doi.org/10.3813/AAA.918139

(C) 2009 S. Hirzel Verlag/European Acoustics Association. The definitive publisher-authenticated version is available online at http://www.ingentaconnect.com/content/dav/aaua. Readers must contact the publisher for reprint or permission to use the material in any form. 


\begin{abstract}
This paper proposes a criterion to determine if an absorbing porous material can be modeled with the "equivalent fluid" limp model instead of Biot model. The limp model is derived from Biot theory assuming that the porous frame has no bulk stiffness. The proposed criterion offers a practical simplification of the frequency dependent criterion defined previously by the authors: it depends only on the bulk modulus of the frame and on its porosity. Frequency independent critical values, below which the effect of the frame stiffness can be neglected, are determined for the whole considered frequency range $[1-10000 \mathrm{~Hz}]$. The critical values are gathered in charts for different porous thicknesses and two configurations: sound absorption of a porous layer backed by a rigid backing and sound radiation of a plate covered by a porous layer. Its is shown that the derived criterion matches Beranek criterion but it is less restrictive.
\end{abstract}

PACS numbers: 


\section{INTRODUCTION}

This paper proposes a criterion to determine if an absorbing porous material can be modeled with the "equivalent fluid" limp model ${ }^{1-6}$ instead of Biot model ${ }^{7,8}$. This criterion offers a practical simplification of the frequency dependent criterion defined previously by Doutres et al. ${ }^{6}$. The use of the limp model is advantageous in finite element models allowing a great decrease of the calculation cost. The criterion involves the limp model rather than the rigid frame model ${ }^{8}$ because it is less restrictive and can be applied for a porous layer bonded onto a vibrating structure.

The criterion defined by Doutres et al. ${ }^{6}$ is based on a frequency dependent parameter FSI (Frame Stiffness Influence) derived from Biot theory. This parameter characterizes the influence of the frameborne wave on the fluid phase displacement. Critical values of FSI, defining the limit below which a porous material can be considered as "limp", were determined for two sets of boundary conditions and three frequency bands characteristic of the frame behavior.

In the present paper, the identification process is more straightforward taking benefit of the simple variation of the FSI parameter with frequency. A frequency independent parameter called $\mathrm{FSI}_{r}$ is then derived from FSI. Critical values are determined considering the maximum error between Biot and limp modeling in the whole frequency range. They are given for several thicknesses and two configurations: sound absorption of a porous layer backed by a rigid wall and sound radiation of a porous layer bonded onto a vibrating wall. A comparison with other criteria ${ }^{1,11}$ is finally presented.

*Electronic address: nicolas .dauchez@univ-lemans.fr 


\section{DERIVATION OF THE FREQUENCY INDEPENDENT PARAMETER FSI $_{r}$}

\section{A. Porous material modeling}

Biot theory ${ }^{7,8}$ is commonly used to model the deformation of a poroelastic solid saturated by a compressible fluid. This theory states that three waves can propagate in a porous medium: two compressional waves and a shear wave. This work deals with one dimensional applications and only the two compressional waves are considered. These waves are characterized by a complex wave number $\delta_{i}(i=1,2)$ and a displacement ratio $\mu_{i}$. Here, the wave with the subscript $i=1$ propagates mainly in the fluid phase and is referred to as the "airborne" wave. The wave with the subscript $i=2$ propagates mainly in the solid phase and is referred to as the "frameborne" wave.

The limp model is derived from Biot theory, assuming that the frame has no bulk stiffness ${ }^{1-6}$. This model describes the propagation of one compressional wave in an "equivalent fluid" medium that has the bulk modulus of the air in the pores and the density of the air modified by the inertia effect of the solid phase and its interaction with the fluid phase.

The compressional wave is characterized by the wave number $\delta_{\text {limp }}=\omega \sqrt{\tilde{\rho}_{\text {limp }} / \tilde{K}_{f}}$ with

$$
\tilde{\rho}_{\text {limp }}=\phi \frac{\rho_{t} \tilde{\rho}_{f} / \phi-\rho_{0}^{2}}{\rho_{t}+\tilde{\rho}_{f} / \phi-2 \rho_{0}} .
$$

Note that the formulation of $\tilde{\rho}_{\text {limp }}$ has been derived from the simplification of Eq. (33) of reference $^{6}$ and has the form of the one recently proposed by Panneton ${ }^{5}$. In this equation, $\phi$ is the porosity, $\rho_{t}=\rho_{1}+\phi \rho_{0}$ is the total apparent mass of the equivalent fluid with $\rho_{1}$ the density of the porous material and $\rho_{0}$ the density of the interstitial fluid. $\tilde{\rho}_{f}$ and $\tilde{K}_{f}$ are the effective density and the bulk modulus of the fluid phase of the well known "rigid frame equivalent fluid" and account for the visco-inertial and thermal interactions between air and frame $e^{6,8}$. 


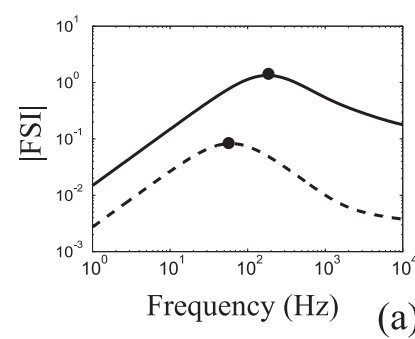

(a)

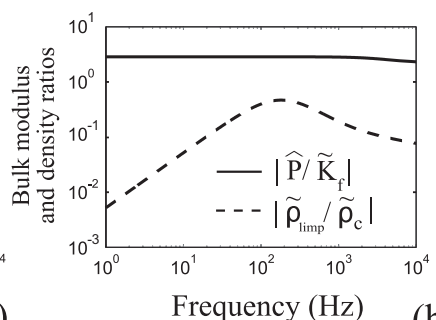

Frequency $(\mathrm{Hz}) \quad$ (b)

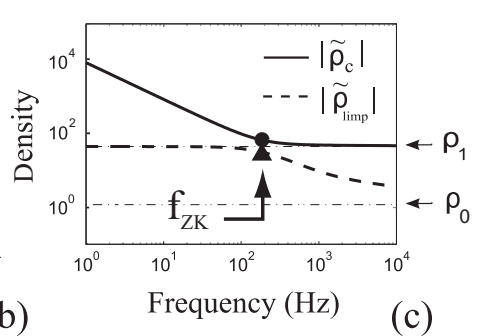

(c)

FIG. 1. (a) Parameter FSI for materials (- -) B and (-) C: $(\bullet)$ are the approximated values at $f_{Z K}$ (see Eq. (6)); (b) ratio of the characteristic Bulk modulus and moduli of complex densities for material C; (c) moduli of complex densities of the two characteristic waves for material C: $(\boldsymbol{\Delta})$ and $(\bullet)$ are the approximated moduli of complex densities at $f_{Z K}$ (see Eq. (4))

\section{B. Frequency behavior of FSI}

The frequency dependent parameter FSI, which characterize the frame influence, is defined in reference ${ }^{6}$. It is derived from Biot theory by assuming that the use of the limp model is possible when the frameborne wave contribution is negligible in the considered application. This condition is observed when the ratio $\mu_{2} / \mu_{1}$ is small compared to $1^{6}$, and can be written as a ratio of two characteristic wave numbers

$$
F S I=\frac{\delta_{\text {limp }}^{2}}{\delta_{c}^{2}}=\frac{\tilde{\rho}_{\text {limp }}}{\tilde{\rho}_{c}} \frac{\widehat{P}}{\tilde{K}_{f}} .
$$

$\delta_{c}=\omega \sqrt{\tilde{\rho}_{c} / \widehat{P}}$ is the wave number of a non-physical wave, called "c" wave, that would propagate in a medium that has the bulk modulus of the frame in vacuum

$$
\widehat{P}=\frac{E(1+j \eta)(1-\nu)}{(1-2 \nu)(1+\nu)}
$$

and the density of the frame in fluid, $\tilde{\rho}_{c}=\rho_{1}-\tilde{\rho}_{12} / \phi$, with $j=\sqrt{-1}, E$ the Young's modulus, $\eta$ the loss factor, $\nu$ the Poisson ratio of the frame and $\tilde{\rho}_{12}$ the inertial coefficient which accounts for the interaction between inertial forces of the solid and fluid phases together with viscous dissipation ${ }^{8}$.

Figure 1(a) presents the FSI for two materials which can be found in automotive or building applications: material $\mathrm{B}$ is a soft fibrous material and material $\mathrm{C}$ is a polymer foam 
with a stiff skeleton (Table I). Note that the Young's modulus of the skeleton, that is kept constant over the considered frequency range, is higher for material C. As a consequence, figure 1(a) shows that the amplitude of the FSI is always higher for material $\mathrm{C}$ for a given frequency. It also appears that both FSI curves exhibit a bell shape.

Considering now only foam C, figure 1(b) shows that this evolution is not explained by the the bulk modulus ratio $\left|\widehat{P} / \tilde{K}_{f}\right|$ that varies slowly, but with the density ratio $\left|\tilde{\rho}_{\text {limp }} / \tilde{\rho}_{c}\right|$ that exhibits a bell shape. At low frequencies, the two phases are strongly coupled by viscous forces: the limp wave density $\tilde{\rho}_{\text {limp }}$ is equal to $\rho_{1}$ while the "c" wave density is greatly superior (see Fig. 1(c)). At higher frequencies, both densities decrease due to the reduction of viscous forces against inertial forces: the "c" wave density tends to $\rho_{1}$ and the limp wave density tends to the fluid density $\rho_{0}$. In between these two asymptotic behaviors, $\tilde{\rho}_{l i m p}$ and $\tilde{\rho}_{c}$ are close and their ratio reaches a maximum. This occurs at the frequency defined by Zwikker and $K_{o s t e n}{ }^{10}, f_{Z K}=\phi^{2} \sigma / 2 \pi \rho_{1}$, that indicates the frequency above which the inertial forces are higher to the viscous ones.

TABLE I. Measured properties of materials B and $\mathrm{C}^{6}$.

\begin{tabular}{lcc}
\hline \hline Porous & B & C \\
\hline Airflow resistivity: $\sigma\left(\mathrm{kN} \mathrm{s} / \mathrm{m}^{4}\right)$ & 23 & 57 \\
Porosity: $\phi$ & 0.95 & 0.97 \\
Tortuosity: $\alpha_{\infty}$ & 1 & 1.54 \\
Viscous length: $\Lambda(\mu \mathrm{m})$ & 54.1 & 24.6 \\
Thermal length: $\Lambda^{\prime}(\mu \mathrm{m})$ & 162.3 & 73.8 \\
\hline Frame density: $\rho_{1}\left(\mathrm{~kg} / \mathrm{m}^{3}\right)$ & 58 & 46 \\
Young's Modulus at $5 \mathrm{~Hz}: E(\mathrm{kPa})$ & 17 & 214 \\
Structural loss factor at $5 \mathrm{~Hz}: \eta$ & 0.1 & 0.115 \\
Poisson's ratio: $\nu$ & 0 & 0.3 \\
\hline \hline
\end{tabular}




\section{Derivation of the frequency independent parameter $\mathbf{F S I}_{r}$}

The frequency independent parameter $\mathrm{FSI}_{r}$ is derived from the maximum value of FSI over the whole frequency range. According to Fig. 1, this maximum can be approximated from the densities of both limp and "c" waves expressed at $f_{Z K}$. Assuming that the density of air $\rho_{0}$ is negligible compared with the one of the porous material $\rho_{1}$, these densities are given by

$$
\tilde{\rho}_{c}\left(f_{Z K}\right) \approx \rho_{1}(1-j / \phi), \tilde{\rho}_{l i m p}\left(f_{Z K}\right) \approx \rho_{1} \frac{(1-j \phi)}{\left(1+\phi^{2}\right)} .
$$

These values are presented on Fig. 1(c) for material C: $(\boldsymbol{\Delta})$ for $\tilde{\rho}_{l i m p}\left(f_{Z K}\right)$ and $(\bullet)$ for $\tilde{\rho}_{c}\left(f_{Z K}\right)$.

At $f_{Z K}$, the bulk modulus of the air in the pores is close to its isothermal value: $\tilde{K}_{f}\left(f_{Z K}\right) \approx$ $P_{0}=101.3 \mathrm{kPa}$. Hence, the modulus of the maximum FSI at $f_{Z K}$ is given by

$$
\left|\mathrm{FSI}\left(f_{Z K}\right)\right| \approx \frac{|\widehat{P}|}{P_{0}} \frac{\phi}{1+\phi^{2}} .
$$

It is noticeable that this parameter is no more function of the frequency parameter $\omega$. In this sense, it is considered as a frequency independent parameter even if $|\widehat{P}|$ may vary slowly with frequency for polymer foam. For most of sound absorbing materials, $\phi$ is close to 1 and Eq. (5) simplifies as

$$
\mathrm{FSI}_{r}=\frac{|\widehat{P}|}{2 P_{0}}
$$

defining the frequency independent parameter $\mathrm{FSI}_{r}$ used in the following. $\mathrm{FSI}_{r}$ is easy to calculate and requires only the knowledge of the bulk modulus of the frame $\widehat{P}$. The two parameters $\mathrm{FSI}_{r}$ and $f_{Z K}$, given in Table II for materials $\mathrm{B}$ and $\mathrm{C}$ are presented with black points in Fig. 1(a).

\section{FREQUENCY INDEPENDENT CRITICAL VALUES AND APPLICATION}

In the previous section, the frequency independent parameter $\mathrm{FSI}_{r}$ which characterizes the intrinsic frame influence has been introduced. The next step is to identify the critical 
TABLE II. Criterion parameters of materials B and C.

\begin{tabular}{lcc}
\hline \hline Material & $\mathrm{B}$ & $\mathrm{C}$ \\
$f_{Z K}(\mathrm{~Hz})$ & 57 & 186 \\
$\mathrm{FSI}_{r}$ & 0.08 & 1.43 \\
\hline \hline
\end{tabular}
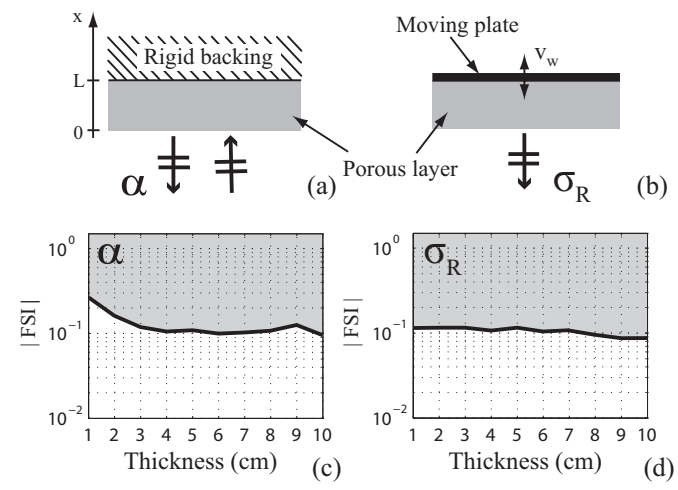

FIG. 2. Design of the two configurations and evolution of the FSI critical values (bold line) as function of the thickness: (a,c) absorption coefficient, (b,d) radiation efficiency.

values under which the limp model can be used instead of Biot model. A similar approach to reference ${ }^{11}$ has been used. The procedure is fully described in reference ${ }^{6}$.

The influence of the frame vibration on the porous material behavior depends on the stiffness of the material but also on frequency and on the boundary conditions applied to the porous layer ${ }^{1-6}$. The critical values of FSI are derived for two specific configurations: sound absorption of a porous layer backed by a rigid wall (Fig. 2(a)) and sound radiation of a plate covered by a porous layer (Fig. 2(b)).

For each configuration and porous layer thickness, the critical values of FSI are determined from the difference between the limp and Biot simulations for a wide range of porous materials. The two materials of TAB. II are used and 256 simulated materials are built with random values of the main porous properties (see TAB. III) to have a set representative of usual absorbing materials. For these simulated materials, the porosity is set to 0.97 and corresponds to classical sound absorbing materials. The viscous characteristic length $\Lambda$ is 
derived from the shape factor $M^{9}$ set to unity (cylindrical pores) and the thermal length $\Lambda^{\prime}$ is three times the viscous characteristic length.

For all materials, the difference between the two models is defined as the maximum difference found over every third octave bands in the frequency range $[1-10000 \mathrm{~Hz}]$. This difference is then sorted in terms of FSI: critical values are set when the difference between the two models exceeds the acceptable limit of 0.1 for the absorption coefficient and $3 \mathrm{~dB}$ for the radiation efficiency.

The two charts given in Figs. 2(c) and 2(d) present the FSI critical values determined for the two configurations and thicknesses from 1 to $10 \mathrm{~cm}$. For a given material, the limp model can be used if the $\mathrm{FSI}_{r}$ is under the critical value (white area of the charts) and Biot model should be preferred if $\mathrm{FSI}_{r}$ exceeds the critical value (gray area of the charts). According to Fig. 2, the critical FSI values are similar for the two considered configurations and slightly depend on the porous thickness: critical FSI $\approx 0.1$.

TABLE III. Range of values for the properties of the simulated materials $\left(\mu^{\prime}=1.8410^{-5} \mathrm{~kg}\right.$ $\mathrm{m}^{-1} \mathrm{~s}^{-1}$ is the viscosity of air).

\begin{tabular}{lc}
\hline \hline Air flow resistivity: $\sigma\left(\mathrm{kN} \mathrm{s} / \mathrm{m}^{4}\right)$ & $1-100$ \\
Porosity: $\phi$ & 0.97 \\
Tortuosity: $\alpha_{\infty}$ & $1-2$ \\
Shape factor: $M$ & 1 \\
Viscous length: $\Lambda(\mu \mathrm{m})$ & $\sqrt{8 \alpha_{\infty} \mu^{\prime} / \sigma \phi}$ \\
Thermal length: $\Lambda^{\prime}(\mu \mathrm{m})$ & $3 \Lambda$ \\
Frame density: $\rho_{1}\left(\mathrm{~kg} / \mathrm{m}^{3}\right)$ & $10-90$ \\
Young's Modulus: $E(\mathrm{kPa})$ & $3-200$ \\
Structural loss factor: $\eta$ & $0.01-0.2$ \\
Poisson's ratio: $\nu$ & 0.3 \\
\hline \hline
\end{tabular}


The procedure for determining the porous materials for which the limp model can be used in the frequency range $[1-10000 \mathrm{~Hz}]$ is as follows: (i) the bulk modulus of the frame $\widehat{P}$ has to be determined; (ii) the parameter $\mathrm{FSI}_{r}$ is evaluated by Eq. (6) using the maximum value of $|\widehat{P}|$ in the considered frequency range; (iii) the critical values of FSI are chosen in Fig. 2 according to the studied configuration and the thickness of the porous layer; (iv) FSI $r$ is finally compared to the critical values: the limp model can be used in the whole frequency range if $\mathrm{FSI}_{r}$ is below the critical value.

In the case of material $\mathrm{B}, \mathrm{FSI}_{r}$ is equal to 0.08 (see TAB. II) which is below critical values for all configurations and thicknesses: the limp model can be used. The $\mathrm{FSI}_{r}$ of material $\mathrm{C}$ is equal to 1.43 (see TAB. II) which is above critical values for all configurations and thicknesses: Biot model should be preferred. These predictions agree with the simulations presented in Fig. 4 of reference ${ }^{6}$.

\section{COMPARISON WITH OTHER CRITERIA}

Beranek $^{1}$ first proposed the limp approximation from the poroelastic formulation of Zwikker and Kosten ${ }^{10}$ using the assumption that, for soft materials, the two waves which propagate in the porous medium are decoupled. He also gave a criterion to identify the porous materials for which this assumption can be applied, that is $\left|\widehat{P} / K_{f}\right|<0.05$. Its criterion limit has been obtained from the study of materials having a frame bulk modulus from 28 to $100 \mathrm{kPa}$. Note that the $\mathrm{FSI}_{r}$ given in Eq. (6) is half the Beranek parameter. In the case of the absorption and radiation configurations, the critical value of FSI is found around 0.1 [see Figs. 2(c) and 2(d)] from the study of materials having a frame bulk modulus from 3 to $200 \mathrm{kPa}$. The proposed criterion can thus be written as $\left|\widehat{P} / K_{f}\right|<0.2$. Hence, the main difference with the Beranek criterion is the critical value: the $\mathrm{FSI}_{r}$ criterion being much less restrictive. Indeed, according to TAB. II, the Beranek criterion rejects the use of the limp model for both materials B and C.

Pilon et al. ${ }^{12}$ proposed a criterion to identify if the effect of the $1 / 4$ wavelength frame 
resonance (occurring at frequency $f_{r}$ ) will be apparent in the absorption coefficient. The criterion in based on an empirical parameter called $\mathrm{FAE}=\sigma E / \rho_{1}^{2}$ that is the product of ratios involved in the definition of $f_{r}$ and $f_{Z K}$. FAE is not rigorously applicable with material B having a Young's modulus under the considered lower limit of $30 \mathrm{kPa}^{11}$. In the case of foam $\mathrm{C}$, the criterion based on FAE is consistent with $\mathrm{FSI}_{r}$ criterion.

\section{CONCLUSION}

In this paper, a frequency independent criterion to identify the porous materials which can be modeled with the limp model has been defined. It is based on a parameter derived from Biot model and is shown to be only function of the bulk modulus of the frame $\widehat{P}$ and of the porosity. In the case of porous materials having a porosity $\phi$ close to 1 , it can be reduced

to $\mathrm{FSI}_{r}=|\widehat{P}| / 2 P_{0}$ which is half the parameter proposed by Beranek ${ }^{1}$. For the majority of thicknesses comprised between 1 and $10 \mathrm{~cm}$, the absorption coefficient and the radiation efficiency could be simulated with the limp model instead of Biot model if $\mathrm{FSI}_{r}<0.1$, i.e. if the bulk modulus of the frame is lower than $20 \mathrm{kPa}$, which is less restrictive than Beranek ${ }^{1}$ criterion.

\section{Acknowledgments}

This study was supported in the scope of the CREDO research project co-funded by the European Commission.

\section{References}

1 L. L. Beranek, "Acoustical properties of homogeneous, isotropic rigid tiles and flexible blankets", J. Acoust. Soc. Am. 19(4), 556-568, (1947).

2 K. U. Ingard, Notes on sound absorption technology, (Noise Control Foundation, New York, 1994). 
3 S. Katragadda, H. Y. Lai and J. S. Bolton, " A model for sound absorption by an sound transmission through limp fibrous layers", J. Acoust. Soc. Am. 98, 2977, (1995).

4 O. Dazel, B. Brouard, C. Depollier and S. Griffiths, "An alternative Biots displacement formulation for porous materials", J. Acoust. Soc. Am. 121(6), 3509-3516, (2007).

5 R. Panneton, "Comments on the limp frame equivalent fluid model for porous media", J. Acoust. Soc. Am. 122(6), EL 217-222, (2007).

6 O. Doutres, N. Dauchez, J.M Genevaux and O. Dazel, "Validity of the limp model for porous materials: A criterion based on Biot theory", J. Acoust. Soc. Am. 122(4), 20382048, (2007).

7 M. A. Biot, "The theory of propagation of elastic waves in a fluid-saturated porous solid. I. Low frequency range. II. Higher frequency range", J. Acoust. Soc. Am. 28, 168-191, (1956).

8 J. F. Allard, Propagation of Sound in Porous Media: Modelling Sound Absorbing Materials, (Elsevier Applied Science, London, 1993).

9 D. L. Johnson, J. Koplik and R. Dashen, "Theory of dynamic permeability and tortuosity in fluid-saturated porous media", J. Fluid Mechanics 176, 379-402, (1987).

10 C. Zwikker and C.W. Kosten, Sound absorption materials, (Elsevier applied science, New York, 1949).

11 D. Pilon, R. Panneton and F. Sgard, "Behavioral criterion quantifying the edgeconstrained effects on foams in the standing wave tube", J. Acoust. Soc. Am. 114(4), 1980-1987, (2003).

12 D. Pilon, R. Panneton and F. Sgard, "Frame acoustical excitability : a decoupling criterion for poroelastic materials", EuroNoise, Naples, (2003). 